Sección Agrícola / Agriculture

Artículo de investigación / Research paper

\title{
Estudio poblacional de Steneotarsonemus spinki (Acari: Tarsonemidae) sobre tres variedades de arroz sembradas en diferentes épocas
}

\author{
Population study of Steneotarsonemus spinki (Acari: Tarsonemidae) on three varieties rice sowing in different times
}

\section{JOSÉ ANTONIO RUBIANO-RODRÍGUEZ1, TATIANA SÁNCHEZ-DORIA² y RODRIGO TOFIÑO-RIVERA ${ }^{3}$}

\begin{abstract}
${ }^{1}$ Ph. D. Corporación Colombiana de Investigación Agropecuaria AGROSAVIA, CI La Selva km 7 vía Las Palmas, Rionegro, Cesar, Colombia, jrubiano@ agrosavia.co, https://orcid.org/0000-0002-2145-4162. ${ }^{2}$ I. A. Corporación Colombiana de Investigación Agropecuaria AGROSAVIA, CI Motilonia km 5 vía Becerril-Codazzi, Cesar, Colombia, tsanchezd@agrosavia.co, https://orcid.org/0000-0001-5797-770X. ${ }^{3}$ I. A. Corporación Colombiana de Investigación Agropecuaria AGROSAVIA, CI Motilonia km 5 vía Becerril-Codazzi, Cesar, Colombia, rtofiNo@agrosavia.co, https://orcid.org/0000-0002-7438-4532.
\end{abstract}

Autor para correspondencia: José Antonio Rubiano-Rodríguez. Ph. D. Corporación Colombiana de Investigación Agropecuaria AGROSAVIA, CI La Selva $\mathrm{km} 7$ vía Las Palmas, Rionegro, Cesar, Colombia, jrubiano@ agrosavia.co, https://orcid.org/0000-0002-21454162

Citación sugerida / Suggested citation RUBIANO-RODRÍGUEZ, J. A.; SÁNCHEZDORIA, T.; TOFIÑO-RIVERA, R. 2019. Estudio poblacional de Steneotarsonemus spinki (Acari: Tarsonemidae) sobre tres variedades de arroz sembradas en diferentes épocas. Revista Colombiana de Entomología 45 (1): e7809 https://doi.org/10.25100/socolen.v45i1.7809

Recibido: 13-jun-2016

Aceptado: 20-ene-2019

Publicado: 30-ago-2019

Revista Colombiana de Entomología ISSN (Impreso): 0120-0488

ISSN (En línea): 2665-4385

http://revistacolombianaentomologia.univalle.edu.co/

Open access

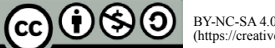

Publicadores / Publishers:

Sociedad Colombiana de Entomología

SOCOLEN (Bogotá, D. C., Colombia)

http://www.socolen.org.co

Universidad del Valle (Cali, Colombia)

http://www.univalle.edu.co/

(C) 2019 Sociedad Colombiana de Entomología - SOCOLEN y Universidad del Valle - Univalle
Resumen: Entre los factores limitantes para la producción de arroz (Oryza sativa), se encuentran las plagas y enfermedades, responsables del $35 \%$ de las pérdidas económicas y que exigen la adopción de medidas de control adecuadas con el fin de garantizar mejores índices de producción. El ácaro Steneotarsonemus spinki es una de las plagas que en los últimos años ha generado pérdidas en el sector arrocero. El Instituto Colombiano Agropecuario, declaró en 2005 la presencia de la enfermedad conocida como "vaneamiento de la espiga", la cual afecta la calidad del grano y la productividad del cultivo hasta en un $60 \%$, generando pérdidas económicas para los agricultores. S. spinki, hace parte del complejo de organismos que causan el vaneamiento. Por lo anterior el objetivo de este trabajo fue evaluar la dinámica poblacional de $S$. spinki en diferentes épocas de siembra en un centro de investigación de Corpoica en Agustín Codazzi, Cesar, Colombia. Para lo cual, durante 2014 se realizaron siembras cada $20+/-3$ días, de tres variedades diferentes de arroz, para un total de siete siembras en un diseño de bloques completos al azar en franjas fijas con cuatro repeticiones. A los 60 días después de emergencia de cada siembra, se procedió a tomar cuatro plantas por repetición por variedad en cada muestreo, haciendo cinco muestreos durante la etapa de cultivo. En la unidad de entomología del CI Motilonia se cuantificó el número de ácaros en las vainas de las tres últimas hojas de la macolla central de cada planta. Las poblaciones de $S$. spinki, superaron los 100 individuos por planta en algunos muestreos, la variedad FED 733 presentó la mayor población del ácaro y la variedad FED 2000 las menores. Según los resultados las poblaciones de $S$. spinki, aumentan en el segundo semestre del año y en etapas fisiológicas avanzadas del cultivo. Aparentemente las poblaciones del ácaro no influyen sobre los rendimientos de las variedades de arroz evaluadas.

Palabras clave: Dinámica, vaneamieto, plagas, macollas.

Abstract: Pests and diseases are among the most important limiting factors for rice (Oryza sativa) production, as they are responsible for $35 \%$ of economic losses. The adoption of adequate control measures in order to ensure better production rates is required. The Steneotarsonemus spinki mite is a pest that has generated many losses in the rice sector in recent years. The Colombian Agricultural Institute declared in 2005 that the presence of the disease known as "vain grain", which affects grain quality and crop productivity up to 60 $\%$, generated economic losses for farmers. S. spinki, is part of the complex of organisms that cause "vain grain". Therefore the objective of this work was to evaluate the population dynamics of S. spinki in different sowing dates in the Agrosavia investigation center of Agustín Codazzi, Cesar, Colombia. For this objective, during 2014 three different rice varieties were sown every $20+/-3$ days, for a total of seven plantings in a complete block design sown at random in fixed strips with four replications. At 60 days of emergence after each sowing, four plants were taken per replicate per variety at each sampling, with a total of five samplings being performed during the growth stage. In the CI Motilonia entomology unit, the number of mites were counted in the pods of the last three leaves of the central till of each plant. The populations of $S$. spinki exceeded 100 individuals per plant in some samples, the variety FED 733 presented the largest population of the mite, and the variety FED 2000 represented the smallest one. According to the results, the populations of $S$. spinki increased in the second semester in advanced physiological stages of the crop. Mite populations appear to have no effect on the yields of the rice varieties evaluated.

Keywords: Dynamics, vain grain, pest, caespitose.

\section{Introducción}

Existen factores bióticos y abióticos que pueden llegar a afectar la producción de arroz, dentro de los primeros están las diferentes plagas. En el cultivo el ácaro Steneotarsonemus spinki (Smiley, 1967) puede causar reducciones en la productividad 
del arroz e inducir el aumento de las aplicaciones de productos de síntesis química para su control. Lo cual conlleva a incrementar los costos de producción, los riesgos de contaminación del ambiente y de los agricultores (Toro 2013). En 1997 se reportó por primera vez su presencia en Cuba sobre el cultivo del arroz (Ramos y Rodríguez 1998), y a partir de este momento inicia su distribución por el Caribe, Centro y sur América (Rodríguez et al. 2009). En Colombia fue registrado por el ICA en 2005 y mediante la resolución 1195 del mismo año fue declarada la emergencia fitosanitaria en todo el territorio nacional por la presencia de los ácaros $S$. spinki y $S$. furcatus (De León) en arroz (ICA 2005). Mediante acciones de vigilancia fitosanitaria y diagnóstico vegetal y por información de productores de semilla se detectó la presencia de $S$. spinki en Casanare, Tolima, Huila y Norte de Santander, y de $S$. furcatus en el Tolima (Toro y Mesa 2014). Además, tanto $S$. spinki y $S$. furcatus han sido reportados sobre cultivos de arroz en los departamentos de Córdoba, Sucre, Bolívar, Magdalena, Cesar, Norte de Santander, Valle del Cauca, Tolima, Huila y Meta (Toro 2013). El hospedero natural preferido por $S$. spinki, es Oriza sativa L. (Lo y Ho 1979; Jiang et al. 1994), pero Lo y Ho (1979) han encontrado este ácaro sobre 70 especies de plantas incluyendo malezas que crecen con el arroz. Sanabria y Aguilar (2005) revelan que S. spin$k i$ en Costa Rica y Panamá usa como hospederos alternos el arroz silvestre Oryza latifolia Desv. En India fue colectado de Cyndon dactylon, (Poaceae), Cyperus sp. (Cyperaceae), Palla (Cyperaceae), Schoenoplectus articulates (L.) (Rao y Prakash 1996, 2002; Central Rice Research Institute 2006).

A nivel mundial $S$. spinki es considerado el ácaro de mayor importancia económica en el cultivo del arroz (Rao et al. 2000; Almaguel et al. 2000; Xu et al. 2001), al causar pérdidas que pueden alcanzar hasta el $90 \%$ de las cosechas, como en Taiwán (Cheng y Chiu 1999), China (Jiang et al. 1994), India (Ou et al. 1977), Cuba (Ramos y Rodríguez 2000), República Dominicana (Ramos y Rodríguez 2001), Panamá (García 2005). En Colombia el sector arrocero se ha visto afectado negativamente a partir del segundo semestre del 2009 debido a diferentes factores, uno de estos es el daño causado por el ácaro $S$. spinki que aunque reportado en los cultivos del país, no había incidido aún en las producciones del grano (Fedearroz 2010). Este problema se acentúa por las temperaturas extremas debidas al cambio climático que afectan la fisiología de la planta (Castilla et al. 2010). Los factores antes mencionados junto a la escasez de agua en los distritos de riego han ocasionado problemas en el llenado del grano, que en algunos casos disminuye la producción hasta en un $50 \%$ (Fedearroz 2010).

Los atributos biológicos de $S$. spinki, tales como la capacidad de desarrollar rápidamente resistencia a plaguicidas, reducido tamaño; por lo cual es muy difícil de detectar; capacidad de sobrevivir en condiciones adversas, reproducción partenogenética y adaptación rápida a nuevos hospederos como las gramíneas, entre otros; lo ubican como una amenaza en los sistemas arroceros (Toro 2013). Estudios realizados en Cuba reportan que una hembra puede colocar hasta 78 huevos a lo largo de su vida, teniendo de 48 a 55 generaciones en un año (Correa-Victoria 2006). Sus poblaciones pueden alcanzar niveles superiores a 300 ácaros por centímetro cuadrado. La longevidad de los adultos varía de 7 a 15 días dependiendo igualmente de la temperatura (Almaguel y Botta 2005). Existen estudios que demuestran la estrecha relación entre los factores abióticos con incrementos de la población del ácaro, tal como temperaturas superiores a 27 ${ }^{\circ} \mathrm{C}$, aumento en las precipitaciones y en la humedad relativa (Cabrera et al. 2003; Quirós y Camargo 2011; Pérez y Duque 2012).

Los daños causados por $S$. spinki pueden ser directos, debido a la alimentación del ácaro en el interior de la vaina de la hoja y en las espigas en formación e indirectos por la inyección de toxinas y la diseminación de microorganismos, especialmente hongos (Santos et al. 2004). Las plantas afectadas presentan granos vanos, parcialmente llenos, manchados, curvatura anormal del pedúnculo de las panículas y necrosis en el interior de las vainas (Ramos y Rodríguez 2000; Almaguel et al. 2002). Las poblaciones de S. spinki dependen del estado fisiológico del cultivo (Ramos y Rodríguez 2001; Rodríguez et al. 2009; Pérez y Duque 2012; Toro y Mesa 2014). Por ejemplo en Cuba las poblaciones fueron bajas durante el estado de macollamiento y se multiplicaron durante la floración, pero el máximo se obtuvo durante el llenado del grano y decreció con la maduración del mismo de lechoso a blando. De igual manera las épocas de siembra también influyen en la densidad poblacional del ácaro, siendo más bajas durante el primer semestre que en el segundo, periodo durante en el cual las poblaciones son muy altas y los daños mayores (Leyva Fernández et al. 2003). Además, la variedad de siembra tiene un papel importante sobre el ataque del ácaro. En Colombia se ha concluido que las variedades Fedearroz Victoria I, Oryzica llanos 4 y Fedearroz 275 presentan mayores poblaciones que las variedades Fedearroz 174, Fedearroz 2000 y Fedearroz 60 (Pérez y Duque 2012).

Para establecer un buen manejo integrado de esta plaga, es necesario conocer las fluctuaciones poblacionales de $S$. spinki tanto en los diferentes estados de desarrollo del cultivo como en diferentes épocas de siembra posible. Por lo tanto, esta investigación, se desarrolló con el objetivo de identificar y evaluar la dinámica poblacional del ácaro S. spinki y su efecto en el rendimiento en tres variedades de arroz establecidas en diferentes épocas de siembra en el Caribe seco colombiano.

\section{Materiales y métodos}

El estudio se realizó entre abril y diciembre del 2014, en el lote $\mathrm{N}^{\circ}$ 8A-4 del Centro de Investigación Motilonia, de AGROSAVIA, ubicado en el municipio de Agustín Codazzi (10¹'55.4'N; 73¹3'29.9'O), kilómetro 5 vía a Bucaramanga, Cesar, Colombia. Se realizaron siete siembras cada $20 \pm$ 3 días iniciando en abril de 2014, con las variedades comerciales Fedearroz, 473 (FED 473), Fedearroz 733 (FED 733) y Fedearroz 2000 (FED 2000). En Cada época de siembra las variedades tenían una franja de $500 \mathrm{~m}^{2}$ con cuatro repeticiones de $125 \mathrm{~m}^{2}$ cada una, para un total de $1.500 \mathrm{~m}^{2}$ por época de siembra.

Muestreo. Se inició a partir de los 60 hasta los 100 días después de emergencia (dde) en cada siembra. Se estableció una cuadrícula, ubicada en el centro de cada repetición, de cada variedad, marcando cuatro puntos por cuadricula, evitando el efecto de borde. El recorrido se inició por la derecha, en dirección norte-sur. En cada punto se tomó una macolla incluyendo su sistema radicular, para un total de 16 macollas por variedad y 48 por siembra. A cada muestra se le lavó el sistema radicular, posteriormente se marcó con el nombre de la variedad y el punto de muestreo (1 a 4). Luego se colocaron en un recipiente con agua para ser llevadas a la unidad de entomología 
Tabla 1. Población de Steneotarsonemus spinki en tres muestreos diferentes durante tres épocas de siembra en tres variedades de arroz en Codazzi, Cesar, Colombia.

\begin{tabular}{lcccccc}
\hline & \multicolumn{5}{c}{ Siembra 4 } \\
\cline { 2 - 7 } & \multicolumn{2}{c}{ Muestreo 80 dde } & \multicolumn{2}{c}{ Muestreo 90 dde } & \multicolumn{2}{c}{ Muestreo 100 dde } \\
\cline { 2 - 7 } & Inmaduros & Adultos & Inmaduros & Adultos & Inmaduros & Adultos \\
\hline Fedearroz 473 & $266,6 \mathrm{ab}$ & $0,9 \mathrm{~b}$ & 283,6 & 2,9 & $239,7 \mathrm{a}$ & $8,89 \mathrm{a}$ \\
Fedearroz 2000 & $8 \mathrm{~b}$ & $2,3 \mathrm{~b}$ & 80,1 & 55,9 & $14,7 \mathrm{~b}$ & $1,6 \mathrm{~b}$ \\
Fedearroz 733 & $543,6 \mathrm{a}$ & $20,4 \mathrm{a}$ & 291,8 & 92,6 & $93,5 \mathrm{~b}$ & $5,2 \mathrm{ab}$ \\
\hline \multicolumn{7}{c}{ Siembra 5 } \\
\hline Fedearroz 473 & 117,4 & 2,3 & $223,4 \mathrm{a}$ & $0,3 \mathrm{~b}$ & $122,7 \mathrm{~b}$ & $19,4 \mathrm{~b}$ \\
Fedearroz 2000 & 291,2 & 1,2 & $121 \mathrm{ab}$ & $0,3 \mathrm{~b}$ & $129,2 \mathrm{~b}$ & $3,5 \mathrm{~b}$ \\
Fedearroz 733 & 290,8 & 2,1 & $57,8 \mathrm{~b}$ & $0,7 \mathrm{a}$ & $406,5 \mathrm{a}$ & $160,9 \mathrm{a}$ \\
\hline & \multicolumn{7}{c}{ Siembra 6 } & \\
\hline Fedearroz 473 & 175,9 & $0,56 \mathrm{~b}$ & $220 \mathrm{~b}$ & $17,3 \mathrm{~b}$ & $23 \mathrm{~b}$ & $4,1 \mathrm{~b}$ \\
Fedearroz 2000 & 72,7 & $0,31 \mathrm{~b}$ & $24,3 \mathrm{~b}$ & $0,31 \mathrm{~b}$ & $55,2 \mathrm{~b}$ & $9,7 \mathrm{~b}$ \\
Fedearroz 733 & 207,3 & $19,38 \mathrm{a}$ & $590,8 \mathrm{a}$ & $92,8 \mathrm{a}$ & $1244,1 \mathrm{a}$ & $546,6 \mathrm{a}$ \\
\hline & \multicolumn{7}{c}{ Siembra 7 } \\
\hline Fedearroz 473 & $510,5 \mathrm{~b}$ & $34,4 \mathrm{~b}$ & 569,90 & $197,5 \mathrm{~b}$ & 193,2 & $134,2 \mathrm{ab}$ \\
Fedearroz 2000 & $148 \mathrm{~b}$ & $16,2 \mathrm{~b}$ & 817,4 & $272,7 \mathrm{~b}$ & 190 & $106,9 \mathrm{~b}$ \\
Fedearroz 733 & $2197,7 \mathrm{a}$ & $386 \mathrm{a}$ & 1784,9 & $857 \mathrm{a}$ & 543,9 & $367,7 \mathrm{a}$ \\
\hline
\end{tabular}

dde $=$ días después de emergencia. Cada dato corresponde al promedio de la lectura de 48 vainas. Columnas por siembra con igual letra no difieren estadísticamente $(\mathrm{P}<0,05)$.

del CI Motilonia y hacer el respectivo análisis de la muestra, identificación y conteo de S. spinki. La lectura de la muestra se realizó en las vainas de las tres primeras hojas de la macolla principal, siendo la hoja bandera la número 1 y las dos siguientes 2 y 3 respectivamente. Se cuantificó la población del ácaro presente en cada una de las vainas seleccionadas por variedad, en cada muestreo y época de siembras. Además, se registró la fecha y los dde de cada muestreo. Para el conteo del ácaro, las muestras se revisaron con estereoscopio de luz directa y en cada vaina se contabilizó el número total de ácaros de estados inmaduros de desarrollo (huevo, larvas, ninfa) y adulto. Para el análisis se hizo sumatoria de todos los estados inmaduros. También se estimó el rendimiento de cada variedad en las diferentes épocas de siembra, tomando el rendimiento de un $\mathrm{m}^{2}$, utilizando un marco de $1 \times 1 \mathrm{~m}^{2}$ que era colocado en el centro de cada repetición. La muestra del rendimiento se desgranó mediante el método del golpeteo, posteriormente se limpió con ayuda de un ventilador y finalmente se pesó en una balanza digital.

Análisis de datos. El diseño experimental utilizado fue el de bloques completos al azar distribuidos en franjas fijas. Se rea- lizó análisis de varianza y pruebas de separación de medias (Tukey $0,05 \%$ ) cuando fue necesario con el programa estadístico SAS 9.3 (SAS 2011).

\section{Resultados y discusión}

En las primeras tres siembras entre abril y mayo, no se encontraron poblaciones del ácaro en ninguno de sus estados de desarrollo en las tres vainas evaluadas. Lo anterior es coincidente con lo reportado por Leyva Fernández et al. (2003), quienes indican las siembras de arroz realizadas durante el primer semestre pueden presentar poblaciones bajas o nulas de $S$. spinki comparadas con las del segundo semestre. La presencia del ácaro se evidenció a partir de la siembra cuatro, la cual se efectuo a finales de junio de 2014. Estos resultados indican que el ácaro inicia su infestación sobre las plantas de arroz en el segundo semestre. Lo cual coincide con Leyva Fernández et al. (2003), donde las infestaciones de S. spinki son superiores durante el segundo semestre en los sitios donde se cultiva arroz. Sin embargo, las poblaciones en este estudio fueron bajas en los primeros muestreos en cada siembra (60 y 70 dde). Estos resultados de bajas poblaciones durante las

Tabla 2. Población de Steneotarsonemus spinki en sus diferentes estados sobre tres hojas evaluadas en diferentes siembras de la variedad FED 733 (Codazzi, Cesar, Colombia).

\begin{tabular}{lccccccccc}
\hline & \multicolumn{3}{c}{ Siembra 5 } & \multicolumn{3}{c}{ Siembra 6 } & \multicolumn{3}{c}{ Siembra 7 } \\
\cline { 2 - 9 } & Hoja 1 & Hoja 2 & Hoja 3 & Hoja 1 & Hoja 2 & Hoja 3 & Hoja 1 & Hoja 2 & Hoja 3 \\
\hline Huevos & 84,8 & 75,0 & 63,3 & $320,7 \mathrm{a}$ & $229,8 \mathrm{ab}$ & $103,7 \mathrm{~b}$ & $459,6 \mathrm{a}$ & $450,5 \mathrm{a}$ & $148,4 \mathrm{~b}$ \\
Larvas & 82,6 & 156,6 & 147,4 & $324,5 \mathrm{a}$ & $217,8 \mathrm{ab}$ & $177,4 \mathrm{~b}$ & $389,4 \mathrm{a}$ & $376,3 \mathrm{a}$ & $131,7 \mathrm{~b}$ \\
Ninfas & $67,3 \mathrm{a}$ & $54,8 \mathrm{ab}$ & $23,6 \mathrm{~b}$ & $338,3 \mathrm{a}$ & $249,9 \mathrm{ab}$ & $80,4 \mathrm{~b}$ & $350 \mathrm{a}$ & $342,1 \mathrm{a}$ & $93,6 \mathrm{~b}$ \\
Adultos & $11,1 \mathrm{~b}$ & $105,1 \mathrm{a}$ & $47,6 \mathrm{ab}$ & $346,1 \mathrm{a}$ & $232,2 \mathrm{~b}$ & $82,5 \mathrm{c}$ & $378,5 \mathrm{a}$ & $280,8 \mathrm{a}$ & $94,4 \mathrm{~b}$ \\
\hline
\end{tabular}

Filas en cada siembra con igual letra no difieren estadísticamente $(\mathrm{P}<0,05)$. 
Tabla 3. Población de Steneotarsonemus spinki en sus diferentes estados sobre tres tiempos de muestreo en diferentes siembras de la variedad FED 733 (Codazzi, Cesar, Colombia).

\begin{tabular}{lccccccccc}
\hline & \multicolumn{3}{c}{ Siembra 5 } & \multicolumn{3}{c}{ Siembra 6 } & \multicolumn{3}{c}{ Siembra 7 } \\
\cline { 2 - 9 } & $\mathbf{8 0}$ dde & $\mathbf{9 0}$ dde & $\mathbf{1 0 0}$ dde & $\mathbf{8 0 ~ d d e}$ & $\mathbf{9 0} \mathbf{d d e}$ & $\mathbf{1 0 0}$ dde & $\mathbf{8 0}$ dde & $\mathbf{9 0}$ dde & $\mathbf{1 0 0}$ dde \\
\hline Huevos & $16,7 \mathrm{~b}$ & $75,3 \mathrm{ab}$ & $131,1 \mathrm{a}$ & $90,9 \mathrm{~b}$ & $151,1 \mathrm{ab}$ & $412,1 \mathrm{a}$ & $900,3 \mathrm{a}$ & $0 \mathrm{c}$ & $158,3 \mathrm{~b}$ \\
Larvas & 41 & 211,4 & 134,2 & $74,4 \mathrm{~b}$ & $242 \mathrm{ab}$ & $403,3 \mathrm{a}$ & $682,3 \mathrm{a}$ & $0 \mathrm{c}$ & $215,1 \mathrm{~b}$ \\
Ninfas & $0,2 \mathrm{~b}$ & $4,2 \mathrm{~b}$ & $141,3 \mathrm{a}$ & $42 \mathrm{~b}$ & $197,8 \mathrm{ab}$ & $428,8 \mathrm{a}$ & $615,2 \mathrm{a}$ & $0 \mathrm{c}$ & $170,6 \mathrm{~b}$ \\
Adultos & $0,7 \mathrm{~b}$ & $2,2 \mathrm{~b}$ & $160,9 \mathrm{a}$ & $19,4 \mathrm{~b}$ & $92,8 \mathrm{~b}$ & $548,8 \mathrm{a}$ & $386 \mathrm{a}$ & $0 \mathrm{~b}$ & $367,7 \mathrm{a}$ \\
\hline
\end{tabular}

Filas en cada siembra con igual letra no difieren estadísticamente $(\mathrm{P}<0,05)$. dde $=$ días después de emergencia.

primeras etapas de desarrollo del cultivo han sido reportados por diversos autores, quienes indican que las poblaciones de $S$. spinki generalmente son bajas en estados iniciales de desarrollo de la planta y van aumentando en los estados más avanzados (Rodriguez et al. 2009; Ramos y Rodriguez 2001; Almaguel et al. 2000; Santos et al. 2004). Lo anterior coincide con los resultados de este estudio, donde la población del ácaro aumento en las etapas finales de desarrollo $(80,90 \mathrm{y}$ 100 dde).

Con respecto a las poblaciones de S. spinki registradas por variedad, la variedad Fedearroz 733 tendió a presentar las mayores poblaciones del ácaro tanto en estado adulto como

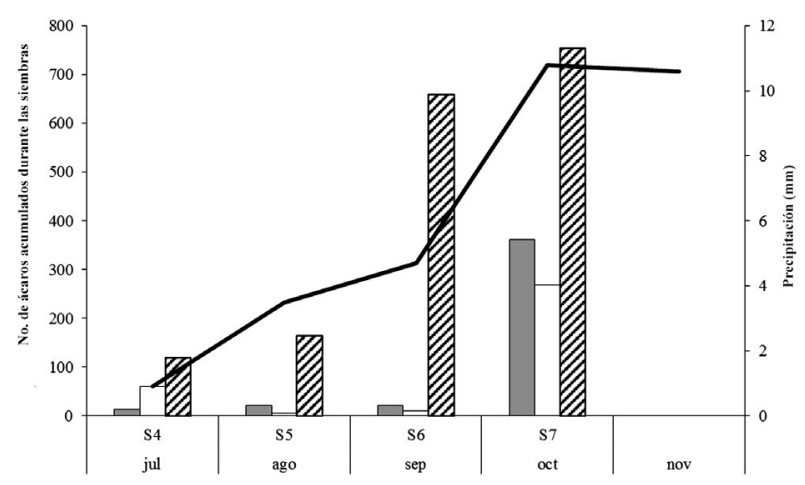

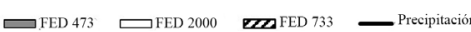

Figura 1. Población acumulada de Steneotarsonemus spinki con respecto a la precipitación promedia mensual del semestre II de 2014 de las tres variedades evaluadas en cuatro épocas diferentes de siembra (Codazzi, Cesar, Colombia).

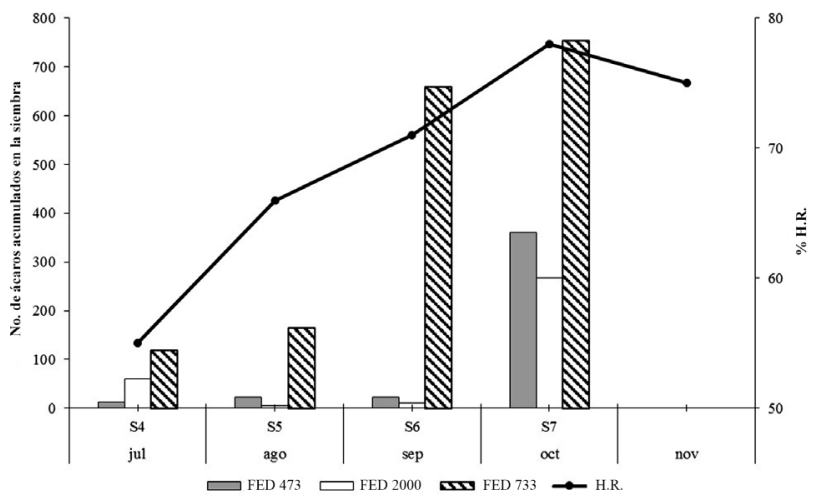

Figura 2. Población acumulada de Steneotarsonemus spinki con respecto a la humedad relativa promedia mensual del semestre II de 2014 de las tres variedades evaluadas en cuatro épocas diferentes de siembra (Codazzi, Cesar, Colombia). inmaduro, siendo significativa a las otras variedades, principalmente con la variedad Fedearroz 2000 que tiende a presentar las menores poblaciones (Tabla 1). Resultados similares se han reportado en Montería, donde estas tres variedades fueron evaluadas junto a otras más (Pérez y Duque 2012). Las poblaciones de $S$. spinki, que llegaron a más de 100 individuos (inmaduros y/o adultos), en algún momento para las tres variedades, son superiores a las reportadas en otros estudios a nivel nacional. Es así como Pérez y Duque (2012) reportan poblaciones de $S$. spinki en promedios de 21,25 y 30 individuos en las variedades FED 2000, FED 473 y FED 733 respectivamente. En otros países como Panamá las poblaciones reportadas por Quirós y Camargo (2011), son similares a las reportadas en este estudio.

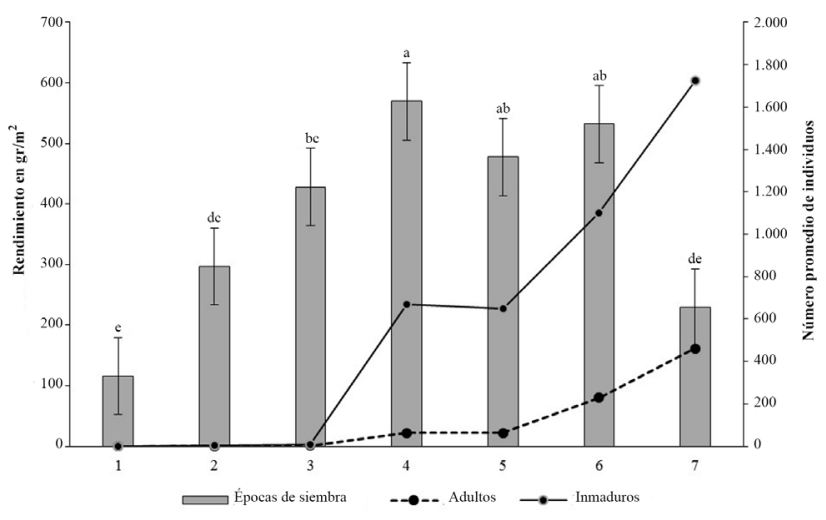

Figura 3. Efecto de la población de Steneotarsonemus spinki sobre rendimiento de arroz en tres variedades de arroz durante siete épocas de siembra en Codazzi, Cesar, Colombia.

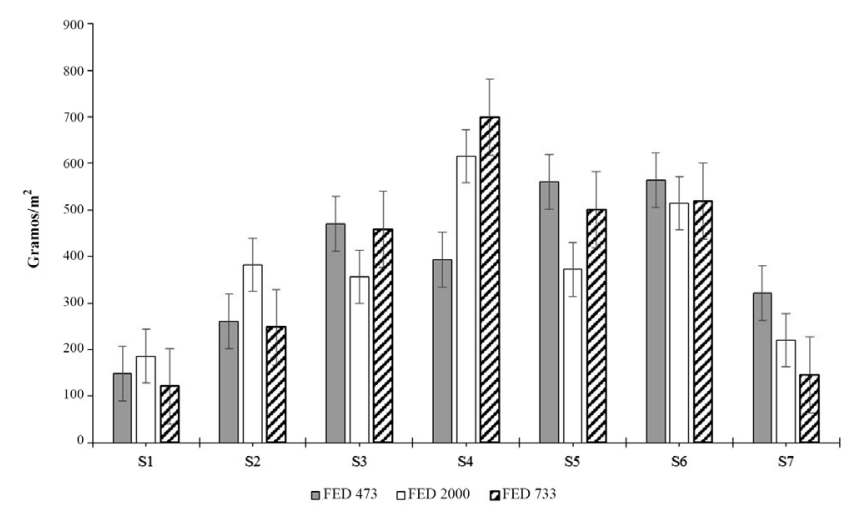

Figura 4. Distribución del rendimiento de tres variedades de arroz durante siete épocas de siembra en Codazzi, Cesar, Colombia. 
Con respecto a la población del ácaro entre las hojas de cada variedad, la población de $S$. spinki en la hoja número uno de la variedad FED 733 fue significativamente superior a la de la hoja número tres, principalmente en las siembras seis y siete en todos los estados de desarrollo del ácaro (Tabla 2). En el muestreo a los 100 dde, esta misma variedad presentó poblaciones superiores en todos los estados del ácaro principalmente en las siembras cinco y seis con respecto al muestreo a los 80 dde (Tabla 3). Las poblaciones del ácaro tanto en estado adulto como inmaduro aumentaron considerablemente cuando aumentó la precipitación (Fig. 1) y la humedad relativa (Fig. 2). Además, la temperatura promedia en la zona de estudio durante el segundo semestre fue superior a $28^{\circ} \mathrm{C}$. Por lo cual se puede inferir que los factores abióticos como precipitación, humedad relativa y temperatura favorecen el incremento de la población de $S$. spinki. Existen reportes que indican cómo el aumento en las precipitaciones, humedad relativa y temperatura superior al $27^{\circ} \mathrm{C}$ en una región donde se cultive arroz favorecen el incremento de la población del ácaro (Santos et al. 2004; Quirós y Camargo 2011; Pérez y Duque 2012). Cabe mencionar que al comparar el rendimiento por $\mathrm{m}^{2}$, nos indica que existen diferencias estadísticas entre las diferentes épocas de siembra. Presentando rendimientos más altos en la época cuatro y los más bajos en la época uno. Sin embargo, los resultados no plantean que las poblaciones del ácaro afecten el rendimiento del cultivo bajo las condiciones estudiadas (Fig. 3). Al parecer estas diferencias en los rendimientos se deben a un déficit hídrico que se presentó durante las primeras siembras.

Con respecto al rendimiento entre variedades, hubo diferencias estadísticas en algunas de las siembras. Es así como en la siembra cuatro, las variedades FED 733 y FED 2000 presentaron rendimientos superiores a la variedad FED 473. Mientras que en la siembra cinco el rendimiento de la variedad FED 473 fue superior a la de la variedad FED 2000. En las otras siembras no hubo diferencia significativa, pero se puede observar la tendencia que tiene la variedad FED 473 a presentar mayores rendimientos en las siembras 3, 6 y 7 (Fig. 4). La inconsistencia de los rendimientos a través de las diferentes épocas de siembra en este experimento, posiblemente se debió al bajo régimen de lluvias durante el periodo de estudio. Además, de algunas fallas en el sistema de riego que se aplicó. Está suficientemente soportado en la literatura que el arroz de riego es un cultivo que necesita de buen suministro de agua (lluvia o riego) para obtener buena producción.

\section{Conclusiones}

Las poblaciones del ácaro $S$. spinki comienzan aumentar sobre las diferentes variedades de arroz cuando el cultivo llega a su etapa de floración (60-70 dde) y durante el segundo semestre del año. Siendo la variedad FED 733 la más susceptible de las tres evaluadas. Los factores abióticos que se presentaron en la zona de estudio favorecen el incremento de la población del ácaro, pero de acuerdo a los resultados no se puede inferir si influye sobre los rendimientos de arroz de riego en la zona de estudio.

\section{Agradecimientos}

Los autores expresan sus agradecimientos al Ministerio de Agricultura el cual financió la investigación a través del con- venio No 1828 con la Corporación Colombiana de Investigación Agropecuaria (AGROSAVIA). A la estación experimental la Victoria de Fedearroz en Montería y al ingeniero Cristo Rafael Pérez por la capacitación en el reconocimiento del ácaro. Al señor Jaime Luis Herrera Villa por su colaboración en el trabajo de campo.

\section{Literatura citada}

ALMAGUEL, L.; BOTTA, E. 2005. Manejo integrado de Steneotarsonemus spinki, Smiley. Resultados de Cuba y transferencia para la región de Latinoamérica y el Caribe. Curso de Posgrado de Ácarología, Introducción a la Acarología Agrícola. La Habana, Cuba. $44 \mathrm{p}$.

ALMAGUEL, L.; HERNÁNDEZ, J.; DE LA TORRE, P. E.; SANTOS, A.; CABRERA, R. I.; GARCÍA, A.; RIVERO, L. E.; BÁEZ, L.; CÁCERES, I.; GINARTE, A. 2000. Evaluación del comportamiento del ácaro Steneotarsonemus spinki (Acari: Tarsonemidae) en los estudios de regionalización desarrollados en Cuba. Fitosanidad 4 (1-2): 15-19.

ALMAGUEL, L.; CABRERA, R. I; HERNÁNDEZ, J.; RAMOS, M.; SANDOVAL, I. 2002. Etimología, biología, ecología y manejo integrado del vaneado de la panícula y pudrición de la vaina del arroz en Cuba. Resultado Científico Premio MINAGRI y de la Academia de Ciencias de Cuba, Septiembre 2002. 245 p.

CABRERA, I. M.; RAMOS, M. L.; FERNÁNDEZ, M. B. 2003. Factores que influyen la abundancia de Steneotarsonemus spinki en arroz, en Cuba. Revista Manejo Integrado de Plagas y Agroecología 69: 34-37.

CASTILLA, L. A.; PINEDA, D.; OSPINA, J.; ECHEVERR, J.; PERAFÁN, R.; GARCES, G.; SIERRA, J.; DIAZ, A. 2010. Cambios climáticos y producciones de arroz. Revista Arroz 58 (489): 5.

CENTRAL RICE RESEARCH INSTITUTE (CRRI). 2006. A new alternate host of rice panicle mite. CRRI Newsletter 27 (3): 10.

CHENG, C. H.; CHIU, Y. I. 1999. Review of changes involving rice pests and their control measures in Taiwan since 1945. Taipei. Plant Protection Bulletin 41 (1): 9-34.

CORREA-VICTORIA, F. 2006. Complejo ácaro-hongo-bacteria en el arroz. Centro Internacional de Agricultura Tropical (CIAT). Cali, Colombia.7 p.

FEDEARROZ. 2010. Evaluación socioeconómica del arroz en Colombia. Disponible en: http://www.fedearroz.com.co/revistanew/arroz488.pdf [Fecha revisión: 15 diciembre 2015].

GARCÍA, M. P. 2005. Vaneamiento y manchado de grano en cultivos de arroz en Panamá. Revista Arroz 53 (455): 10-13.

INSTITUTO COLOMBIANO AGROPECUARIO (ICA). 2005. Resolución No. 001195 de 2005. Diario Oficial, edición 45.892. Miércoles 27 abril de 2005. Bogotá, Colombia. 17 p.

JIANG, P. Z.; XIE, X. J.; CHEN, W. X.; CAO, S. Y.; LIANG, Z. H. 1994. Regularity of incidence of Steneotarsonemus spinki. Guandong. Agricultural Science 5: 37-40.

LEYVA FERNÁNDEZ, Y.; ZAMORA ZAMORA, N.; ÁLVAREZ ÁLVAREZ, E.; JIMÉNEZ, M. 2003. Resultados preliminares de la dinámica poblacional del ácaro Steneotarsonemus spinki. Revista Electrónica Granma Ciencia 7 (1): 1-6.

LO, K. C.; HO, C. C. 1979. Ecological observations on rice tarsonemid mite, Steneotarsonemus spinki (Acarina: Tarsonemidae). Journal of agricultural research of China 28 (3): 181-192.

OU, T.; FANG, H. C.; TSENG, Y. H. 1977. Studies on Steneotarsonemus madecassus Gutierrez of rice. Plant Protection Bulletin 19: 21-29.

PÉREZ, C. R; DUQUE, M. C. 2012. Muestreo y comportamiento del ácaro Steneotarsonemus spinki en el cultivo de arroz en Montería, Colombia. Revista Arroz 60 (497): 31-39.

QUIRÓS, E; CAMARGO, I. 2011. Respuesta de variedades de arroz (Oryza sativa L.) a las poblaciones de Steneotarsonemus spinki Smiley (Acari: Tarsonemidae) en Panamá. Revista de Protección Vegetal 26 (1): 30-39. 
RAMOS, M.; RODRÍGUEZ, H. 1998. Steneotarsonemus spinki Smiley (Acari: Tarsonemidae): Nuevo informe para Cuba. Revista de Protección Vegetal 13: 25-28.

RAMOS, M.; RODRÍGUEZ, H. 2000. Ciclo de desarrollo de Steneotarsonemus spinki Smiley (Acari: Tarsonemidae) en laboratorio. Revista de Protección Vegetal 15 (2): 51-52.

RAMOS, M.; RODRÍGUEZ, H. 2001. Aspectos biológicos y ecológicos de Steneotarsonemus spinki en arroz, en Cuba. Manejo Integrado de Plagas y Agroecología 61: 48-52.

RAO, J.; PRAKASH, A. 1996. Cynodon dactylon (Linn.) Pers. (Graminae): an alternate host of rice tarsonemid mite, Steneotarsonemus spinki Smiley. Journal of Applied Zoological Research 7: 50-51.

RAO, J; PRAKASH, A. 2002. Paddy field weed, Schoenoplectus articulatus (Linn.) Palla (Cyperaceae): a new host of tarsonemid mite, Steneotarsonemus spinki Smiley and panicle thrips, Haplothrips ganglbaureri Schmutz. Journal Applied Zoological Research 13: 174-175.

RAO, P. R. M.; BHAVANI, T. R. M.; RAO, T. R. M.; REDDY, P. R. 2000. Spikelet sterility grain discoloration in Andhra Pradesh, India. International Rice Research Notes 25 (3): 40.

RODRÍGUEZ, H.; MIRANDA, I.; JEAN, L.; HERNÁNDEZ, J. 2009. Comportamiento poblacional de Steneotarsonemus spinki Smiley (Acari: Tarsonemidae) en el cultivo del arroz (Oryza sativa L.). Temas de Ciencia y Tecnología 13 (39): 55-66.

SANABRIA, C.; AGUILAR, H. 2005. El ácaro del vaneo del arroz (Steneotarsonemus spinki L: Tarsonemidae). Boletín Fitosanitario, Ministerio de Agricultura y Ganadería, San José, Costa Rica. 16 p.

SANTOS, R.; NAVIA, D.; CABRERA, R. I. 2004 Steneotarsonemus spinki (Acari: Prostigmata: Tarsonemidae) - uma ameaça para a cultura do arroz no Brasil. Documento 117/ Embrapa Recursos Genéticos e Biotecnologia. Brasilia. 48 p.

SAS. 2011. Institute. SAS/STAT 9.3 Base SAS ${ }^{\circledR} 9.4$ Procedures Guide: Statistical Procedures. SAS Institute.
TORO, S. 2013. Dinámica poblacional y estudio de la incidencia del ácaro Steneotarsonemus spinki Smiley (Acari: Tarsonemidae) sobre el desarrollo fenológico del arroz en Colombia. Tesis de Doctorado en Ciencias Agropecuarias. Universidad Nacional de Colombia Sede Palmira. Cali, Colombia. 125 p.

TORO, S; MESA, N. 2014. Parámetros poblacionales y comportamiento de Steneotarsonemus spinki Smiley (Acari: Tarsonemidae) en el cultivo de arroz. Acta Agronómica 64 (2): 186-193. https://doi.org/10.15446/acag.v64n2.43936

XU, G. L.; WU, H. J.; HUAN, Z. L.; WAN, M. 2001 Study on reproductive characteristic of rice mite, Steneotarsonemus spinki (Acari: Tarsonemidae). Systematic and Applied Acarology 6: 45-49. https://doi.org/10.11158/saa.6.1.7

\section{Origen y financiación}

El escrito es producto de la investigación de un proyecto de la agenda quinquenal 2013-2018 de AGROSAVIA, titulado "Prácticas de manejo seleccionadas para recuperar la productividad del cultivo del arroz en el sistema de producción de riego" donde una de sus actividades fue evaluar la poblaciones de Steneotarsonemus spinki y su efecto sobre la productividad del arroz. El proyecto fue financiado por Ministerio de Agricultura el cual financió la investigación a través del convenio No 1828 con la Corporación Colombiana de Investigación Agropecuaria (AGROSAVIA).

\section{Contribución de los autores}

José Antonio Rubiano-Rodríguez: Supervisión del trabajo en campo y laboratorio, análisis de los datos, escritura de resultados y discusión, revisión y edición del documento final.

Tatiana Sánchez-Doria: Toma de muestras, conteo de la población del acaro, escritura de la metodología.

Rodrigo Tofiño-Rivera: Encargado del manejo agronómico de las parcelas experimentales, toma de datos, escritura de las primeras versiones de la introducción. 